\title{
Improving publishing success at JAMS: contribution and positioning
}

\author{
Robert W. Palmatier ${ }^{1}$
}

Published online: 20 September 2016

(C) Academy of Marketing Science 2016

After finishing my first year as Editor-in-Chief (EIC) of the Journal of the Academy of Marketing Science (JAMS) and accepting more than 40, while rejecting nearly 500, manuscripts, I have realized that there are many good ideas and datasets that never make it through the review process for a specific set of reasons. With this editorial, I describe some of the most common reasons and challenges, offer some insights on how to avoid these problems (or resolve them when they occur), and thereby suggest some guidelines for enhancing contributors' publishing success with JAMS. Historically, we have published about 45-48 papers each year; our editorial objective is to increase this number to 60 articles. In support of this goal, we are pursuing several options, including (1) publishing more editorials and commentaries (e.g., Hamilton 2016; Houston 2016; Moorman 2016), (2) hosting more special issues, (3) increasing efforts to increase widespread recognition of JAMS (e.g., recent addition to Financial Times journal list), and (4) enacting a dedicated push to feature underrepresented, substantive domains (e.g., services, consumer-based strategy) and geographies (e.g., Europe and China), such as through Thought Leaders' Conferences.

Although necessarily simplistic and generalized, the guidance in this editorial may help authors increase their publishing success; it may be especially valuable to early career scholars and researchers submitting to JAMS for the first time. It is my hope that it reduces the rate of desk rejections at JAMS as well. All research domains have their own stylistic require-

Robert W. Palmatier palmatrw@uw.edu

1 Michael G. Foster School of Business, University of Washington, PACCAR Hall, Box 353226, Seattle, WA 98195-3226, USA ments, and consistent with the publication objectives for $J A M S$, this editorial is relevant mainly to strategy and managerially focused empirical articles (representing the majority of the scholarship published in JAMS). I focus specifically on research contributions and positioning - two areas that are critical to a paper's ultimate impact and its likelihood of publication success at JAMS. I also offer nine specific recommendations for authors submitting to JAMS.

I thank Mark Houston, Don Lehman, Mike Brady, and Lisa Scheer for their input in creating this editorial, which also reflects the helpful insights I have gleaned from listening to more experienced journal editors in the many meet-the-editor sessions I have participated in during the past year.

\section{Research contribution: need for tension and moderation}

The top reason offered by reviewers, AEs, and the EIC when rejecting a paper is the lack of a sufficient contribution, relative to extant research. This measure is very difficult to evaluate objectively, but there are a few systematic ways to gauge, and then extend if necessary, any particular paper's contribution to the field. First, authors should develop a literature review table, describing relevant papers in consecutive rows and evaluating each paper across 4-6 pertinent columns (e.g., context, theory base, key moderators, key findings). A good literature review table summarizes the field in which the current paper will be positioned, so it helps the authors recognize their own paper's contribution, as well as communicating this information clearly to the reader. In creating these tables, authors must be critical in their evaluation of their own contributions, relative to extant literature, and offer a compelling discussion that supports the differentiation of their work. Reviewers 
frequently know of specific, less referenced articles that have been published previously and express concerns that a submission offers little incremental contribution above that published work. By creating a formal table, authors can become more critical in their own evaluation while also avoiding unsupported, high-level arguments about their contributions. Finally, a literature review table offers a small contribution on its own, in that readers and future researchers can use it as a well-organized base for building their work.

\section{Recommendation 1: Include a well-structured literature review table and arguments to clarify the paper's incre- mental contribution, relative to extant research.}

Second, authors should find a source of "tension" in their conceptual model that will make their paper interesting and not overly simplistic (Davis 1971). By necessity, most conceptual models focus on just a few focal construct(s) that might be critical for explaining managerially relevant outcomes (which is the basis of most strategy research). However, in too many cases, proposed models contain only beneficial pathways in which the focal construct(s) drive performance, without any offsetting mechanisms included. Identifying and including various pathways is critical to building tension in the model and making the research non-trivial. For example, the premise that targeted email campaigns will lead to incremental customer purchases is likely true, but a model predicting this link offers little tension. Reviewers often criticize such papers for offering little that is new, because the findings are not interesting or surprising. However, if such a model also included the cost to the customer of processing the email or the feeling of being stalked by personally targeted recommendations, the paper would create some tension and increase its contribution. Rather than arguing that targeting emails is "always" beneficial, authors should find and evaluate opposing mechanisms that offer trade-offs and produce a more nuanced model. Another common issue arises when authors argue that some "new" construct is a critical antecedent of an important outcome, without empirically modeling a more complete inventory of existing antecedents. In this case, reviewers cannot evaluate the potential for an incremental contribution, over and beyond the known effects. While adding tension to a model is a powerful and common way to add contribution to a paper, it should be recognized that there are other equally valid approaches.

\section{Recommendation 2: Ensure some tension in the concep- tual model to enhance the paper's contribution and avoid overly simplistic arguments.}

Third, authors typically need to include theory-driven, moderating variables in their conceptual model to achieve a sufficient contribution. If the model is built to include tension, the obvious moderators are constructs that will "turn on and off" the opposing pathways and ultimately determine when each pathway will dominate. In an ideal case, the same theory used to predict the main effect mechanisms can also identify potential moderating effects. Moderating variables that operate on one pathway, and are consistent with theory, help undermine alternative explanations. Less desirable moderators would operate in the same direction on all pathways; in this case, they cannot differentiate among opposing mechanisms. Individual difference or other variables that might help in theory testing but are not under a manager's control also are relatively less desirable as moderators in JAMS. It is very difficult to publish a main effect-only strategy paper in a premier journal, because any such model will be highly susceptible to alternative explanations (e.g., common method variance), unrealistic in terms of ignoring boundary conditions, and unhelpful to managers who want to increase their strategic effectiveness.

\section{Recommendation 3: Include theoretically derived, man- agerially controllable moderators in the conceptual model to achieve sufficient contributions.}

Fourth, the data and methods need to be powerful enough that they produce believable results, instead of allowing the contributions to be discounted. Reviewers often mentally add to and subtract from a paper's contribution according to the strength of its data and methods. For example, theoretically interesting, well-supported main effects offer limited contributions if all the constructs come from the same survey respondents, because they risk common method variance (CMV; Podsakoff et al. 2003). Since CMV reduces the power to find significant interaction effects, moderating hypotheses are not undermined by the CMV in survey data and thus offers more potential contribution. The ideal data and method for each paper depends on its conceptual model and research problems, but some consistent characteristics help ensure that a manuscript makes a contribution that can address unique questions and offer more robust results. For example, papers with longitudinal data, objective performance measures (sales, profits, purchase behaviors), field experiments, or measures from multiple perspectives (customer, salesperson, firm) have significantly more apparent potential to provide contribution. I am surprised at how often reviewers bring up the strength of the data; papers with strong data simply get more revision opportunities. In contrast, papers that rely on MTurk and student samples or that measure all their constructs with data from a single respondent tend to signal limited contributions, independent of their theory or conceptual model, because reviewers have less confidence in the robustness of the results. At JAMS, a main effects-only model tested with a single survey likely will be desk rejected, because of 
its small chance of making it through the review process and offering a significant contribution.

\section{Recommendation 4: Devote extra effort to ensuring the data and method are robust enough to prevent the pa- per's contribution from being discounted, due to a lack of confidence in the validity of the results.}

Fifth, to enhance the contribution of an existing paper or help push the paper over the publication hurdle during the review process, authors may want to consider including another, complementary study that helps overcome a significant weakness in the initial study or adds another layer of related contribution. For example, an event study showing the effect of a phenomenon on firms' stock price may have good external validity but provides little insight into the underlying causal mechanisms. Adding an experiment that can demonstrate the underlying mechanisms, with strong internal validity, would be a good complement and enhance the paper's overall contribution. Other potential solutions include (1) adding a strong review or conceptual aspect, (2) generating post hoc elasticity analyses or other analyses to quantify the magnitude of the effect, or (3) augmenting the paper with other data (cases, qualitative interviews) that provide support or insights into the phenomena of interest. However, in these efforts, authors take on the added responsibility of ensuring that the multiple studies and data fit together in a compelling fashion and tell a cohesive story. A grouping of several, unrelated studies, each with a limited contribution, would not be well received in the review process.

\section{Recommendation 5: Consider adding a complementary study or new data to achieve a sufficient contribution for publication.}

\section{Positioning: the small things matter}

Poor positioning is another cause for rejection from JAMS. Positioning comprises many, diverse elements, some of which may seem trivial to authors. But they make far more sense when considered in the context of a typical reviewer's evaluation process as well as a future reader's impression. Accordingly, to present my suggestions regarding positioning, I take a temporal perspective. Many readers and reviewers use heuristics to help them form a general impression about the value of a paper, before beginning their detailed reading. If these signals are mostly negative, they almost unavoidably begin with a negative predisposition and look for ways to support this judgment (i.e., confirmation bias). Individual reviewers consider different factors of course, but some signals are commonly used by reviewers, as well as future readers. I provide these ideas and recommendations to help authors position their work and communicate their message clearly to readers.

Each journal has conventions and norms that should be followed, the most obvious of which is the basic formatting and style of the journal. Each journal establishes certain stylistic preferences, related to the headings, references, and structure. Each submitted paper should match the formatting and feel of the journal where it is submitted; to do otherwise is, in my opinion, research malpractice. When starting to write, authors should identify three or four recent papers from the target journal, most similar in their research approach (e.g., data, methods, approach), that they can use as a stylistic template for structuring their work. In general, if authors are unwilling to reformat a paper to submit it to a new journal, after being rejected at another outlet, why should reviewers spend time reviewing the paper and giving detailed advice? Furthermore, the paper should be grounded in literature previously published in that journal or journals similarly positioned, such as Journal of Marketing (JM) for JAMS. There is no rule or quota for citing papers from the targeted journal, nor should there be, but failing to build on extant research that has appeared in the target journal or related journals suggests that the paper may not be a good fit for the outlet. In effect, authors are saying that no previously published work in these journals is relevant to their paper, which the review team generally considers a concern. At JAMS in particular, consumerbased strategy papers that offer no link to prior research published in $J M$ or $J A M S$, but in which the authors assert their managerial contribution to marketing strategy, prompt skepticism among reviewers.

Overall, consider how the implicit messages sent by seemingly inconsequential elements discussed in this editorial can undermine your ability to communicate the central points of your research. Performing these items by no means guarantee publication, but rather is a minimum hurdle that better allows a paper to be judged on its merits.

\section{Recommendation 6: Ensure that the paper looks, sounds, and feels like other recent papers in the targeted journal.}

Second, figures, tables, and other non-textual elements carry extraordinary weight as communication tools. Readers seek ways to understand the paper's big picture rapidly; they often turn first to the figures and tables to understand what the paper offers, before they begin reading. Strategy research traditionally features a boxes-and-arrows model, including all hypotheses and their numbers, the data sources and collection times, and other features that can help the reader, which can be especially effective at communicating the big picture. In my own reviews, I often print out the model and results tables and refer to them as I read. The tables and figures also must be able to stand alone, exclude acronyms, and use notes to 
offer additional explanations that might be needed to help readers interpret the graphic element. It is authors' responsibility to help readers by labeling all lines rather than using legends, ensuring that the visuals can help tell the story, and providing exhibits of high quality - as close as possible to the quality in published articles.

\section{Recommendation 7: Use sufficient, high-quality figures,} tables, and graphics to help position the paper and aid the reader in understanding its key messages.

Third, the introduction is the first text (or maybe second, after the abstract) that a reviewer reads in detail. My sense is that many reviewers have developed their general recommendation for the paper by the end of the introduction; this assessment even can determine the amount of attention they devote to the overall review. Of course, a positive first impression can be lost if the paper offers up poor data, methods, or other issues, but a negative first impression is difficult to change following a poor introduction. The introduction is where authors sell their work to readers. Although introductions vary somewhat with the approach used for the research, they consistently need to be concise and clear in stating the paper's contributions and operational details (theory, data, methods). Unlike a fiction author writing a thriller, a strategy scholar will face rejection if the paper leaves too many unanswered questions over too many pages. Acknowledging the availability of alternative approaches, here I outline a classic structure for positioning a strategy paper in its introduction:

- Identify the domain and state the paper's objective: To begin, demonstrate the importance of the central study domain, possibly using statistics that signal the prevalence of the issue or some insights from the popular or business press. Discuss extant approaches to this issue and why they are less than satisfactory. The final sentence of this paragraph should state the focus or objective of the paper, offering a logical conclusion to the opening paragraph.

- Conceptual approach: Provide a summary of general theory and conceptual approaches to achieving the paper's objective. This second paragraph should answer key questions: what theoretical lens is being used, how the paper will define its central constructs, and which mediating mechanisms might appear in the model (research tension).

- Empirical approach: Present the empirical approach for testing the conceptual model outlined in Paragraph 2. This third paragraph should address points such as the number of studies, sample characteristics, and general methods adopted. Include a concise explanation of why the chosen research design is appropriate for testing the focal model, especially in papers that present multiple studies.
- Paper contributions: Outline the paper's contributions, with a separate paragraph for each unique contribution. Each paragraph should adopt a parallel structure: describe the contribution, foreshadow the key results that support this contribution, and outline why this specific contribution is important. To establish importance, authors can use post hoc analyses of the managerial impact (median split, elasticity analyses), as well as note how the findings help explain previous anomalies in academic research or the business press and how these results could direct managers' actions.

Recommendation 8: Make the introduction a powerful overview of the paper's positioning and contribution, to motivate readers to invest time in reading the rest of the paper.

Fourth, constructs, headings, and hypotheses need to aid the reader and be consistent throughout. I recommend listing the headings and hypotheses in separate documents when drafting a manuscript, so authors can consider them all together and edit them accordingly and consistently. The purpose of headings is to help the reader navigate the paper, by providing information in a parallel structure. Even a very complex paper can be approachable if it contains well-designed headings. If readers get lost or confused about where the research logic is going, they often skip ahead and simply comment that the paper lacks clear logic or support. Hypotheses are even more critical, from a statistical testing standpoint, but also as a concise summary of the paper's predictions. Many readers use the hypotheses to understand the big picture, especially for papers that do not contain a graphic of the conceptual model. Hypotheses thus should feature a parallel structure, be clearly written, and use consistent terminology, with all constructs clearly defined in the paper and their operationalizations described in detail in the methods section. Finally, authors should ensure that they use consistent terminology for their focal constructs and other important terms (e.g., wholesaler, customer centricity), while avoiding unfamiliar or undefined acronyms (ideally not using more than one new acronym in the paper).

Recommendation 9: Make the constructs, headings, and hypotheses consistent throughout the paper.

\section{Conclusion}

These nine recommendations provide general guidelines that I hope will improve the success of authors who submit their work for publication consideration at JAMS. They are not specific requirements of course; each paper needs to be adapted 
to the specific research question it seeks to address. Many other elements of academic writing also are crucial to a paper's impact. Yet contribution and positioning are the root causes for many of the rejections I have observed in my first year as EIC at JAMS. Such contribution and positioning issues often cause a paper to be rejected immediately, with little opportunity for revision; other issues can be addressed more readily through the review process. I hope these recommendations also improve the quality of the reviews that authors receive, in that papers that match these guidelines will better engage the review team and likely lead to even more insightful feedback.

This editorial discusses many structural and writing items that authors can readily address, but this attention should not undermine the authors' efforts focused on selecting interesting problems and phenomena. In the next issue of JAMS, Gerard Tellis (2016) offers his insights into these important issues in an editorial titled, "Interesting and impactful research: on phenomena, theory, and writing." His editorial is accompanied with commentaries by John Hauser (2016) and Rajan Varadarajan (2016) on the same theme, offering three different perspectives to a topic that I feel is absolutely critical to generating high impact reach. These three papers provide insight into the creative side of academic research as a necessary balance to this editorial's focus on the more mundane and mechanistic details.

Although review and conceptual papers are not the focus of this editorial, JAMS is expanding its capacity as an outlet for such papers in marketing. Many other scholarly areas have a rich tradition and receptive publication outlets for dedicated review papers (empirical meta-analyses and non-empirical review papers), which can help consolidate research insights and provide a platform for the next wave of research in a theoretical, substantive, or methodological domain. I appeal to interested scholars to contact me directly with proposals; I am willing to provide presubmission guidance for such ambitious works, to increase their likelihood of publication success.

Robert W. Palmatier

Editor-in-Chief

\section{References}

Davis, M. (1971). That's interesting! Towards a phenomenology of sociology and a sociology of phenomenology. Philosophy of the Social Sciences, 1(4), 309-344.

Hamilton, R. (2016). Consumer-based strategy: using multiple methods to generate consumer insights that inform strategy. Journal of the Academy of Marketing Science, 44(3), 281-285.

Hauser, J. (2016). Phenomena, theory, application, data, and methods all have impact. Journal of the Academy of Marketing Science. doi:10.1007/s11747-016-0498-1.

Houston, M. (2016). Is "strategy" a dirty word? Journal of the Academy of Marketing Science, 44(5), 557-561.

Moorman, C. (2016). Celebrating marketing's dirty word. Journal of the Academy of Marketing Science, 44(5), 562-564.

Podsakoff, P., MacKenzie, S. B., Lee, J.-Y., \& Podsakoff, N. P. (2003). Common method biases in behavioral research: a critical review of the literature and recommended remedies. Journal of Applied Psychology, 88(5), 879-903.

Tellis, G. (2016). Interesting and impactful research: on phenomena, theory, and writing. Journal of the Academy of Marketing Science. doi:10.1007/s11747-016-0499-0.

Varadarajan, R. (2016). Musings on interesting and impactful theory and research. Journal of the Academy of Marketing Science. doi:10.1007 /s11747-016-0500-y. 